\title{
The Effect of DA-9701 in Opioid-induced Bowel Dysfunction of Guinea Pig
}

\author{
Zahid Hussain, Kwang Won Rhee, Young Ju Lee, and Hyojin Park* \\ Department of Internal Medicine, Gangnam Severance Hospital, Yonsei University College of Medicine, Seoul, Korea
}

\section{Background/Aims}

Opioid induced bowel dysfunction (OIBD) is associated with decreased gastrointestinal (GI) propulsive activity due to intake of opioid analgesics. DA-9701, a novel prokinetic agent formulated with Pharbitis Semen and Corydalis Tuber has promising effects on GI motor function. Therefore, we aim to evaluate the prokinetic effects of DA-9701 in an OIBD model of guinea pig.

\section{Methods}

The ileal and distal colon muscle contraction in presence of different doses of DA-9701, morphine, and combination (morphine + DA9701) was measured by tissue bath study. The prokinetic effect of DA-9701 was assessed by charcoal transit and fecal pellet output assay in an OIBD model of guinea pig.

\section{Results}

DA-9701 significantly increased the amplitude and area under the curve of ileal muscle contraction, while there was insignificant effect on the distal colon compared to the control. The maximal amplitude of ileal muscle contraction was acquired at a concentration of $10 \mu \mathrm{g} / \mathrm{mL}$ of DA-9701. In contrast, morphine significantly decreased the amplitude of ileal and distal colon muscle contraction compared to the control. Morphine delayed both upper $(P<0.01)$ and lower $(P<0.05) \mathrm{Gl}$ transit, and delayed $\mathrm{Gl}$ transit was restored by the administration of DA-9701. Morphine induced reduction of contractility was significantly ameliorated by addition of DA-9701 in both ileal and distal colon muscles.

\section{Conclusions}

DA-9701 significantly increased the amplitude of contraction of the ileal muscle, however the distal colon muscle contraction was insignificant. Additionally, it restored delayed upper and lower GI transit in an OIBD model of guinea pig, and it might prove to be a useful candidate drug in a clinical trial for OIBD.

\section{(J Neurogastroenterol Motil 2016;22:529-538)}

Key Words

DA-9701; Gastrointestinal transit; lleum; Morphine; Opioid

Received: December 8, 2015 Revised: January 15, 2016 Accepted: January 27, 2016

(a) This is an Open Access article distributed under the terms of the Creative Commons Attribution Non-Commercial License (http://creativecommons. org/licenses/by-nc/4.0) which permits unrestricted non-commercial use, distribution, and reproduction in any medium, provided the original work is properly cited.

${ }^{*}$ Correspondence: Hyojin Park, MD, PhD

Department of Internal Medicine, Gangnam Severance Hospital, Yonsei University College of Medicine, 211 Eonju-ro, Gangnamgu, Seoul 06273, Korea

Tel: +82-2-2019-3318, Fax: +82-2-3463-3882, E-mail: hjpark21@yuhs.ac 


\section{Introduction}

Morphine is an opioid analgesic drug and most commonly used in the treatment of malignant as well as functional and chronic gastrointestinal (GI) pain. Although opioids are globally prescribed by physicians as an analgesic, constipation is a common and persistent side effect induced by this opioid. ${ }^{1,2}$ The constipation induced by opioids are commonly referred to as opioid induced bowel dysfunction (OIBD) and it has significant adverse effects on patient's quality of life. ${ }^{3-5}$ The opioid induced constipation is predominantly mediated by gastrointestinal $\mathrm{mu}(\mu)$ opioid receptors. ${ }^{6}$ High density $\mu$ opioid receptors are mostly found in neurons of myenteric, submucosal plexus, and the immune cells in lamina propria. ${ }^{7}$ Morphine activates $\mu$ opioid receptors in the enteric nervous system, which in turn inhibits the normal release of excitatory and inhibitory neurotransmitters that coordinates motility. ${ }^{5,89}$ Inhibition of excitatory neural pathways depresses peristaltic contractions, and on the other hand the blockade of inhibitory neural pathway increases GI muscle activity, elevates resting muscle tone, spasm and nonpropulsive motility patterns. ${ }^{5}$ Hence, $\mu$ receptor activation severely interrupts the rhythmic contractions and is responsible for delayed gastric emptying and slowing of the intestinal transit. ${ }^{8,9}$ Activation of opioid receptors inhibits water and electrolyte secretion into the gut lumen and increases fluid absorption from the intestine, and blood flow in the gut wall. ${ }^{10}$ As a result, the stool remains in the gut lumen for a longer time, therefore more fluid is reabsorbed and the stool becomes hard and dry., ${ }^{5,10}$ The above effects are also associated with opioid inhibition of secretomotor neurons in the epithelium of the gut. Firing of secretomotor neurons evokes secretion of $\mathrm{H}_{2} \mathrm{O}$, electrolytes, and mucus. Morphine acts to hyperpolarize and silence action potential discharge by secretomotor neurons. ${ }^{9}$ The suppression of firing in the secretomotor neuronal pool reduces secretion, and results in lowered liquidity of small and large intestinal contents and drier harder stool in large intestine. ${ }^{11}$

There is currently no approved therapy in the specific management of OIBD, though laxatives are the most commonly used. However, efficacy can be limited and most of them act slowly then abruptly, and also tolerability is poor which makes it difficult to control defecation in everyday life. ${ }^{12}$ Therefore, there is a need for a treatment regimen that has faster onset of action against opioid induced constipation than current laxatives. DA-9701 is a newly formulated prokinetic agent obtained from extracts of Pharbitis Semen and Corydalis Tuber. ${ }^{13-16}$ Both of these plants have been used in oriental traditional medicine for the treatment of GI disorders.
Pharbitis Semen, the seed of Pharbitis nil Choisy, has been used as a folk medicine for its analgesic effects on abdominal disorders. Corydalis Tuber, the root of Corydalis yahusuo W.T. Wang, has been used as a folk medicine for its analgesic and anti-ulcer effects. ${ }^{17-19}$ DA-9701 has shown affinity for the dopamine (D) $)_{2}, 5$-hydroxytryptamine $(5-\mathrm{HT})_{4}, 5-\mathrm{HT}_{1 \mathrm{~A}}$, and $5-\mathrm{HT}_{1 \mathrm{~B}}$ receptors. ${ }^{13,11,15}$ It has antagonistic effects on the $\mathrm{D}_{2}$ receptors and agonistic effects on the $5-\mathrm{HT}_{4}, 5-\mathrm{HT}_{1 \mathrm{~A}}$ and $5-\mathrm{HT}_{1 \mathrm{~B}}$ receptors. ${ }^{14,15,20}$ It has been reported that DA-9701 can improve delayed gastric emptying and may be effective as a prokinetic agent. ${ }^{14}$ Accordingly, DA-9701 is considered as a complex motility agent, which can be a solution to resolve the various symptoms of opioid induced constipation. Therefore, the aim of this study was to evaluate the effects of DA-9701, a novel prokinetic agent in the OIBD model of the guinea pig.

\section{Materials and Methods}

\section{Animals Handling and Preparation}

Adult male Hartley guinea pigs (250-350 g, Orient Bio, Inc, Seoul, Korea) were acclimated to their holding room (temperature controlled at $21 \pm 1^{\circ} \mathrm{C}, 50 \pm 10 \%$ humidity, and 12-hour light/ dark cycle commencing at 7:00 AM) for at least a day prior to surgery. The guinea pigs were deprived of food, but not water for a 24hour period before experiments. The guinea pigs were stunned by a blow on the head and exsanguinated by severing the carotid arteries just before the laparotomy to harvest segments of the ileum or distal colon. The ileum was harvested $5 \mathrm{~cm}$ proximally to the cecum, while the distal colon was harvested $5 \mathrm{~cm}$ proximal to the anus. All experiments were conducted in accordance with the Guide for the Care and Use of Laboratory Animals provided by the Animal Laboratory Ethics Committees of the Department of Laboratory Animal Medicine, Medical Research Center, Yonsei University College of Medicine. This study was approved by the institutional animal care and use committee (IACUC) of Yonsei University College of Medicine with IRB protocol number 2013-0157.

\section{Drugs}

The following drugs and chemicals were used; charcoal (Sigma, St. Louis, MO, USA), barium sulfate (Tae Joon Pharmaceuticals, Seoul, Korea), and DA-9701 (Dong-A, Pharmaceuticals, Seoul, Korea), morphine (Myungmoon Pharm Co, Seoul, Korea). These were freshly prepared for every experiment by dissolving each compound in isotonic sodium chloride solution. 


\section{Experimental Design 1: Effect of DA-9701 on lleal and Colonic Muscle Motor Function}

\section{Tissue bath study}

The ileal and distal colon muscle was removed and cut into 3-mm wide and 10-mm long fragments and the lumen flushed clean using Krebs-Henseleit solution which was maintained at $37^{\circ} \mathrm{C}, \mathrm{pH} 7.4$ and saturated with $95 \% \mathrm{O}_{2}$ and $5 \% \mathrm{CO}_{2}$ in a chamber. The circular muscle was fixed and connected to independent tension transducers (BIOPAC TSD 105; BIOPAC Systems, Inc, Santa Barbara, CA, USA). The preparation was set up and allowed to equilibrate for at least 60 minutes after $1 \mathrm{~g}$ resting tension. Circular muscle activity was quantified by means of a computerized integration procedure (BIOPAC MP 100; BIOPAC Systems, Inc). Drugs were added to the bath solution for 5 minutes without washing between successive changes in concentration. An independent tissue bath study was performed using different concentrations of DA-9701 (1, 5, and $10 \mu \mathrm{g} / \mathrm{mL})$ in order to analyze its effect on ileal and colonic muscle contraction. The maximal amplitude and area under the curves (AUC) of the ileal and distal colon muscle contraction were obtained to evaluate the effects of drugs on GI motility.

\section{Experimental Design 2: Effect of Morphine on lleal and Colonic Muscle Motor Function}

\section{Tissue bath study}

In order to create an experimental model of OIBD, different doses of morphine $(0.1,0.3,1,3,10$, and $30 \mu \mathrm{M})$ was examined for its effect on ileal and distal colon muscle contraction. The most effective dose concentration of morphine was selected to study the effect of DA-9701 in OIBD model.

\section{Charcoal transit assay}

The effect of morphine on upper GI transit was tested by the charcoal transit assay in fasted guinea pigs. Guinea pigs received a vehicle $(0.9 \%$ normal saline) and different concentrations of morphine $(0.3,0.5$, and $1 \mathrm{mg} / \mathrm{kg})$ via an orogastric cannula. After 30 minutes, vehicle ( $0.9 \%$ normal saline) was administered via the orogastric cannula, followed by $2.5 \mathrm{~mL}$ of a charcoal mixture after another 30 minutes wait. Upper GI transit was evaluated as the migration of charcoal mixture from the pylorus to the most distal point of migration, and expressed as a percentage (\%) of charcoal migration $(\mathrm{cm})$ to the length of total small intestine $(\mathrm{cm}) 10$ minutes after injection of the charcoal mixture.

\section{Fecal expulsion study}

Similarly, the fecal pellet output assay was performed in order to test the effect of morphine on lower GI transit of the guinea pig. The fecal pellet output was measured in non-fasted guinea pigs receiving an intragastric administration of the vehicle $(0.9 \%$ normal saline) and different concentrations of morphine $(0.3,0.5$, and 1 $\mathrm{mg} / \mathrm{kg}$ ). After 30 minutes, the vehicle ( $0.9 \%$ normal saline) was administered via the orogastric cannula. Each guinea pig was placed into an individual experimental cage, and both weight and number of fecal pellets produced were measured and recorded. The result of 6 hours of cumulative fecal pellet output was measured for each guinea pig.

\section{Experimental Design 3: Effect of DA-9701 on Morphine Induced OIBD Model}

\section{Tissue bath study}

The effect of different doses of DA-9701 (1, 5, 10, and 20 $\mu \mathrm{g} / \mathrm{mL})$ in the presence of $1 \mu \mathrm{M}$ morphine was recorded by a tissue bath study.

\section{Charcoal transit assay}

The effect of DA-9701 on upper GI transit was tested by the charcoal transit assay in the morphine induced OIBD model of the guinea pig. Guinea pigs received vehicle $(0.9 \%$ normal saline $)$ and morphine $1 \mathrm{mg} / \mathrm{kg}$ via the orogastric cannula. After 30 minutes, vehicle (0.9\% normal saline) and different doses of DA-9701 were administered via the orogastric cannula, followed by $2.5 \mathrm{~mL}$ of a charcoal mixture after another 30 minutes wait. Upper GI transit was evaluated in the same manner as mentioned above.

\section{Fecal expulsion study}

The fecal pellet output assay was performed in order to test the effects of the prokinetic agent DA-9701 on lower GI transit in the morphine induced OIBD model. The fecal pellet output was measured in non-fasted guinea pigs receiving an intragastric administration of vehicle ( $0.9 \%$ normal saline $)$ and morphine $0.3 \mathrm{mg} / \mathrm{kg}$. After 30 minutes, the vehicle ( $0.9 \%$ normal saline) and different doses of DA-9701 were administered via the orogastric cannula and fecal expulsion was measured in the same manner as mentioned above. 


\section{Statistical Methods}

The results for each variable were expressed as a percentage of the control levels. Statistical analysis was performed using repeated measures ANOVA with post hoc comparison. In all test analyses, statistical significance was assigned if $P<0.05$ based on the mean. Values are means with standard error. All data were analyzed using SPSS version 12.0 for Windows software (SPSS Inc, Chicago, IL, USA).

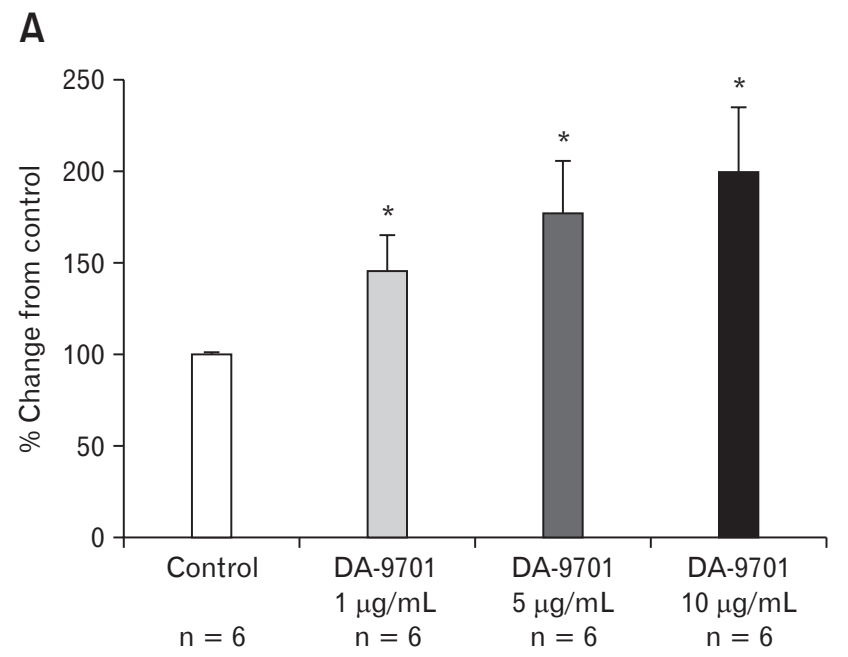

\section{Results}

\section{Effects of DA-9701 on Contraction of Ileal and Distal Colon Muscles}

The degree of ileal muscle contraction was tested at different doses of DA-9701. It significantly increased the amplitude of ileal muscle contraction in a dose dependent manner $(1,5$, and 10 $\mu \mathrm{g} / \mathrm{mL}$ ) compared to the control (Fig. 1A). At a concentration

B

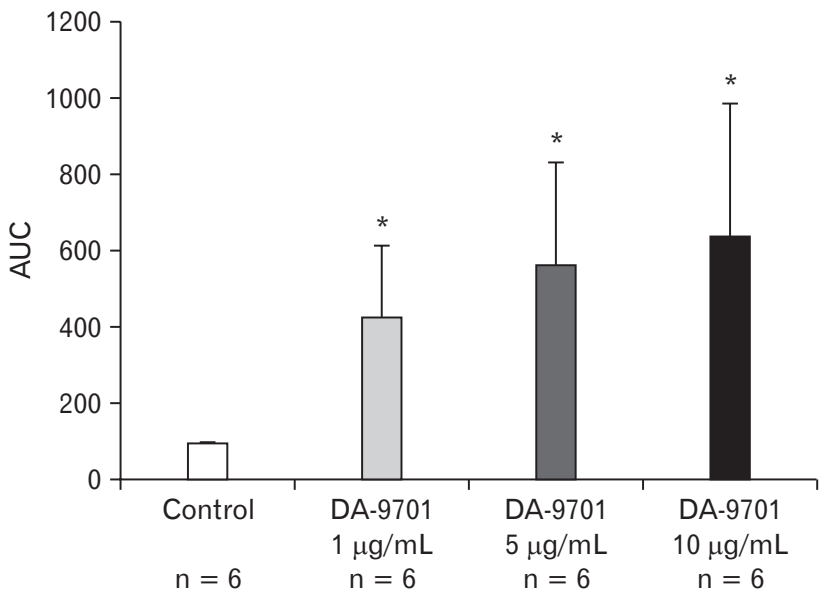

Figure 1. Effect of DA-9701 on contraction of the ileal muscle. DA-9701 increased maximal amplitude and area under the curve in a dose dependent manner $(1-10 \mu \mathrm{g} / \mathrm{mL})$. (A) The percent change of amplitude from control ( $\left.{ }^{*} P=0.010\right)$. (B) The percent change of under the curve (AUC) from control $\left({ }^{*} P=0.010\right)$ Values are represented in mean \pm SEM.

A

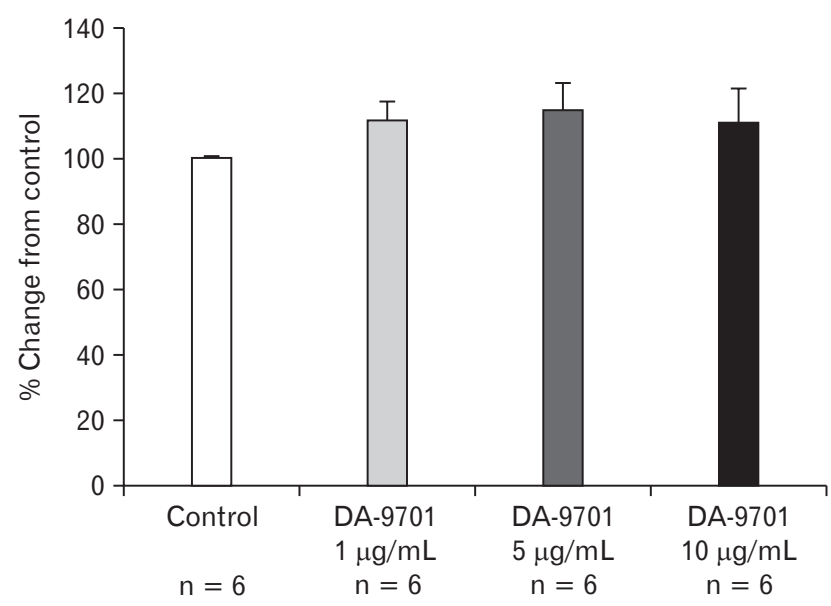

B

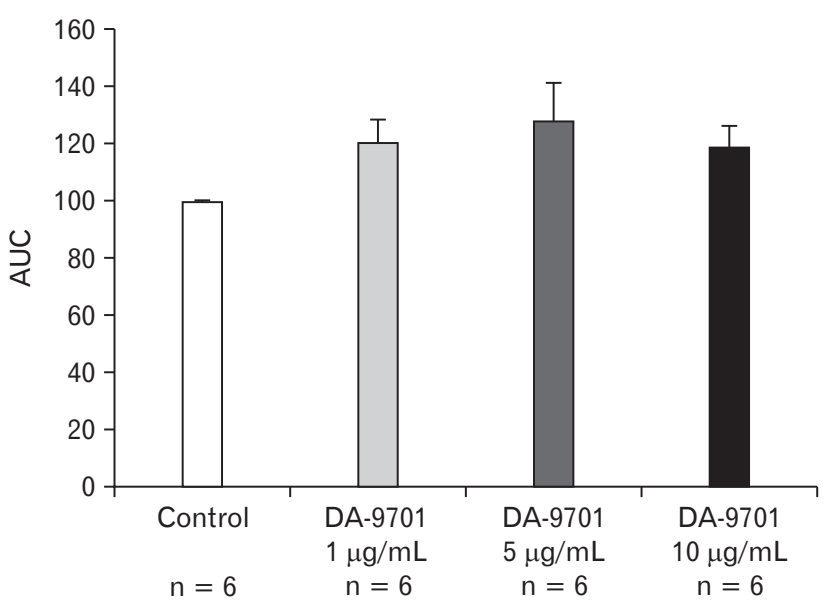

Figure 2. Effect of DA-9701 on contraction of the distal colon muscle. DA-9701 increased amplitude and under the curve (AUC) compared to that of control. Values are represented in mean \pm SEM. 
of $10 \mu \mathrm{g} / \mathrm{mL}$ of DA-9701, the amplitude of the contraction was maximal $(P=0.010)$. As shown in Figure $1 \mathrm{~B}, \mathrm{AUC}$ of the ileal muscle contraction also significantly increased in a dose dependent manner with the highest peak recorded at $10 \mu \mathrm{g} / \mathrm{mL}$ of DA-9701. Similarly, we tested the effects of DA-9701 on the contraction of the distal colon muscle. However, there was insignificant increase in the amplitude and AUC in the presence of different doses of DA-9701 as compared to the control (Fig. 2).

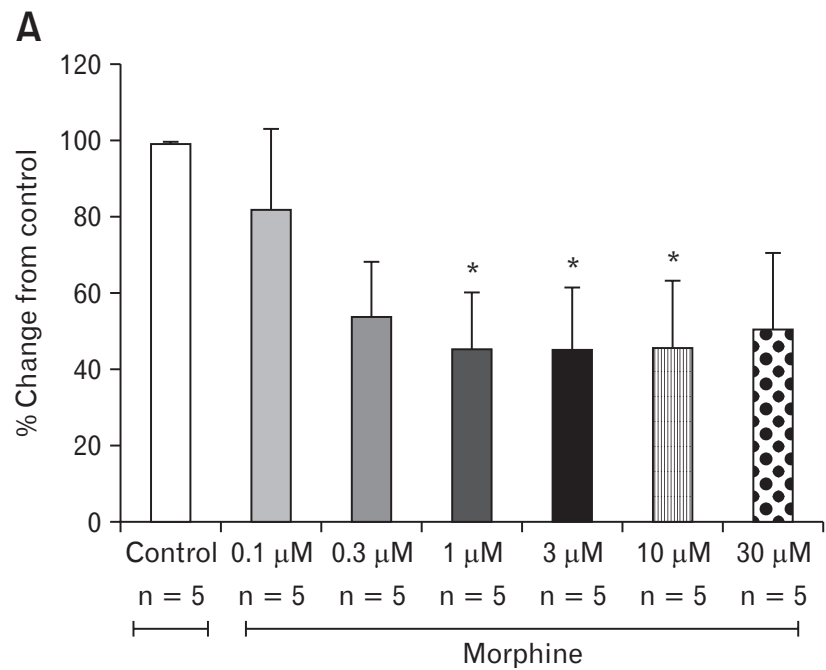

\section{Effect of Morphine on Contraction of lleal and Distal Colon Muscle}

In order to generate an OIBD model, we tested the effect of different concentrations of morphine on the amplitude of contraction in the ileal and distal colon muscles. As shown in Figure 3A, out of 6 different doses of morphine analyzed in this study, 1, 3, and $10 \mu \mathrm{M}$ significantly decreased the amplitude of the contraction in the ileal muscle compared to the control $(P=0.010)$. The ampli-

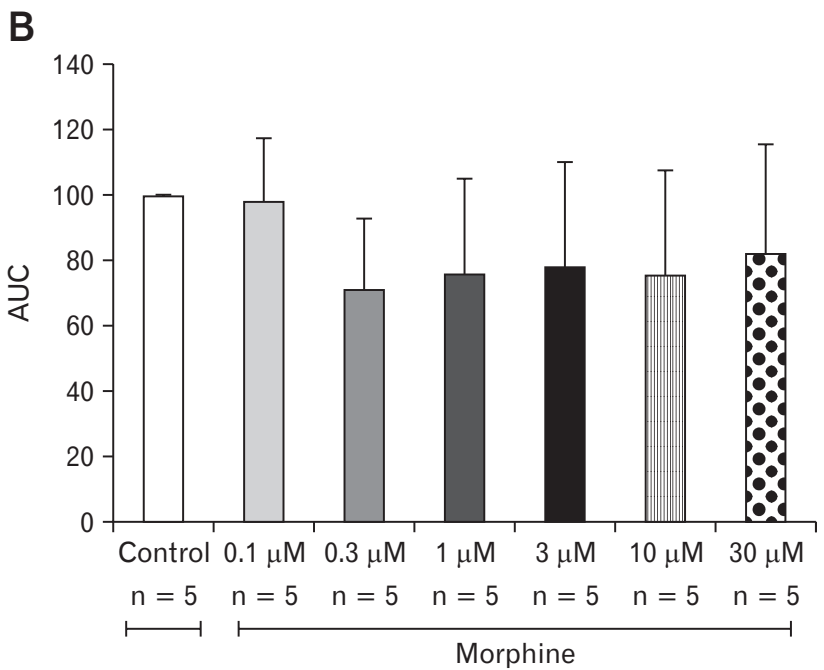

Figure 3. Effects of morphine on contraction of the ileal muscle. Morphine decreased maximal amplitude and area under the curve (AUC). (A) Percent change of amplitude from control $\left({ }^{*} P=0.010\right)$. (B) Percent change of AUC from control. Values are represented in mean \pm SEM.

A

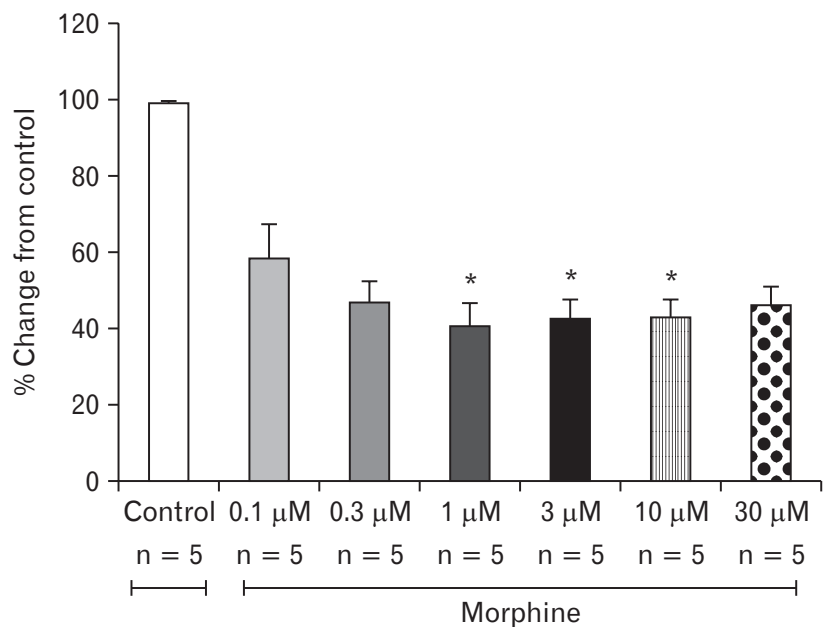

B

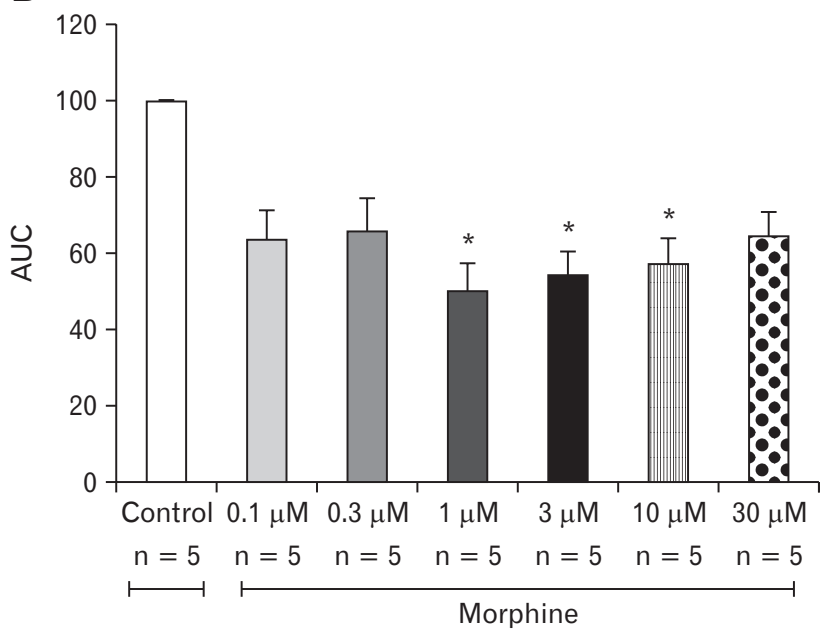

Figure 4. Effects of Morphine on contraction of the distal colon muscle. Morphine decreased maximal amplitude and area under the curve (AUC). (A) Percent change of amplitude from control $\left({ }^{*} P=0.010\right)$. (B) Percent change of AUC from control $\left({ }^{*} P=0.010\right)$ Values are represented in mean \pm SEM. 
tude of ileal muscle contractions were $45.35 \pm 33.64 \%, 45.11 \pm$ $36.86 \%, 45.57 \pm 39.75 \%$ in the presence of 1,3 , and $10 \mu \mathrm{M}$ morphine respectively. In contrast, there was no change in AUC at any dose of the morphine treatment on the ileal muscle (Fig. 3B). In the distal colon muscle, morphine exerts a similar effect on the amplitude as that of the ileal muscle. The doses of 1,3 , and $10 \mu \mathrm{M}$ had respective amplitude values of $41.23 \pm 12.49 \%, 42.97 \pm 11.06 \%$, and $43.58 \pm 9.60 \%$ respectively, and hence significant decrease from the control (Fig. 4A). Similarly, the AUC of the distal colon also significantly decreased at doses of 1,3 , and $10 \mu \mathrm{M}$ (Fig. 4B).

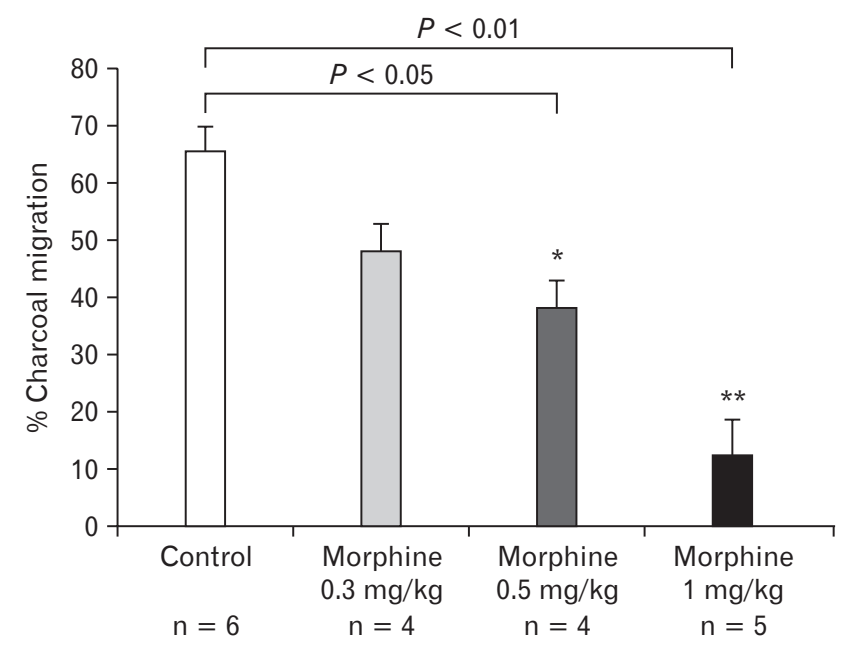

Figure 5. Effect of different doses of morphine on upper gastrointestinal transit in the guinea pig. Values are mean \pm SEM. ${ }^{*} P<0.05$ and ${ }^{*} P<0.01$ in comparison to control.

\section{Effect of Different Doses of Morphine on Upper and Lower Gastrointestinal Transit}

Three different doses $(0.3,0.5$, and $1 \mathrm{mg} / \mathrm{kg})$ of morphine were tested in upper and lower GI transit experiments in order to select the most effective dose for creation of an OIBD model. As shown in Figure 5, the percentage charcoal transit $(\mathrm{cm} / \mathrm{cm} \times 100)$ in the control group was $65.57 \pm 10.83$, while those in the $0.3,0.5$, and $1 \mathrm{mg} / \mathrm{kg}$ morphine treated groups were $48.03 \pm 9.87,38.45 \pm$ 9.20 , and $12.72 \pm 13.21 \%$ respectively. Among the 3 doses tested, dose $1 \mathrm{mg} / \mathrm{kg}$ morphine most significantly delayed the charcoal transit in the upper GI tract compared to the control. In the lower GI transit assay the number of fecal pellets expelled in the control group was $15.22 \pm 15.78$, while those in the $0.3,0.5$, and $1 \mathrm{mg} / \mathrm{kg}$ morphine treated groups were $8.00 \pm 6.78,12.16 \pm 10.55$, and $12.66 \pm 10.83$ respectively. The comparison of the control group with morphine treated groups clearly showed that the dose $0.3 \mathrm{mg} /$ $\mathrm{kg}$ of morphine significantly decreased the cumulative number of fecal pellet output expelled compared to the control (Fig. 6).

\section{Effects of DA-9701 on Morphine Treated lleal and Distal Colon Muscle}

Following testing of the effects of morphine on ileal and distal colon contractility, we selected $1 \mu \mathrm{M}$ morphine as a base dose to analyze DA-9701 prokinetic effects. As shown in Figure 7A, the addition of DA-9701 to base dose $1 \mu \mathrm{M}$ morphine treated ileum increased the amplitude of contraction in a dose dependent manner

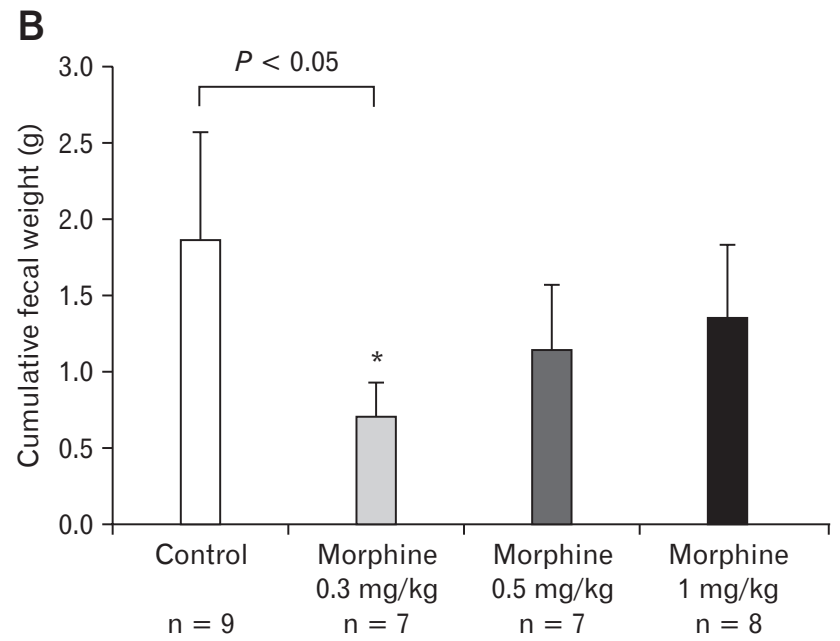

Figure 6. Effect of different doses of morphine on lower gastrointestinal transit in guinea pig. (A) Effect of different concentrations of morphine on cumulative fecal pellet output (FPO). (B) Effect of different concentrations of morphine on cumulative FPO weight (g). Values are mean \pm SEM. ${ }^{*} P<0.05$ in comparison to control. 
(1-20 $\mu \mathrm{g} / \mathrm{mL})$. Ten and $20 \mu \mathrm{g} / \mathrm{mL}$ DA-9701 concentrations significantly restored the amplitude and AUC of ileal muscles treated with morphine $1 \mu \mathrm{M}$ (Fig. 7). Similarly, DA-9701 also restored the amplitude of distal colon contraction and AUC in a dose dependent manner (1-20 $\mu \mathrm{g} / \mathrm{mL}$ ) (Fig. 8). Unlike the ileal muscle, the distal colon showed significantly high contraction only at the maximal concentration.

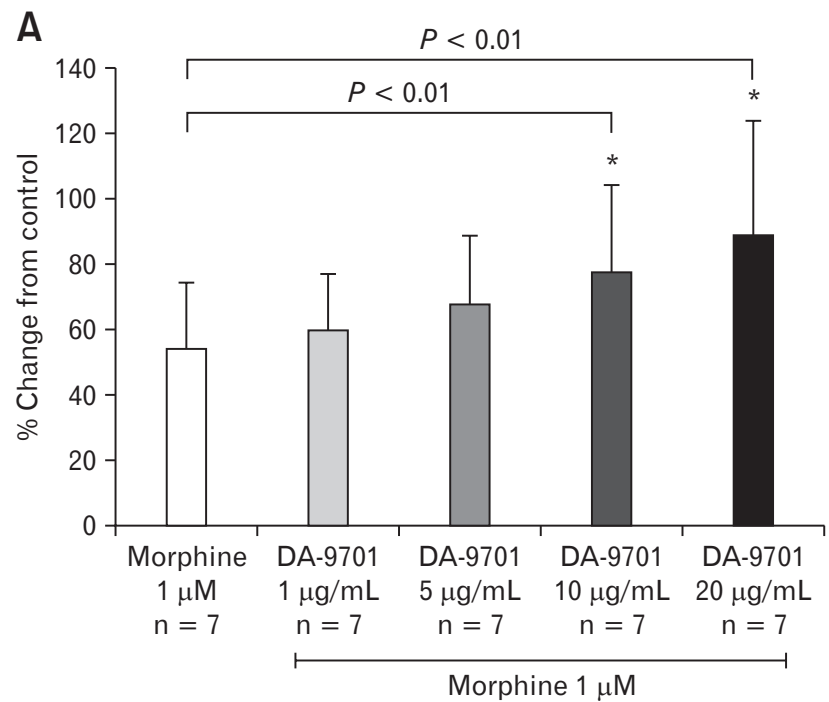

\section{Effects of DA-9701 on the Charcoal Transit in Opioid Induced Bowel Dysfunction Model}

The most effective dose of morphine ( $1 \mathrm{mg} / \mathrm{kg}$ ) was selected in order to induce the experimental model of the OIBD in the guinea pig. The prokinetic effect of different doses $(1,3$, and $10 \mathrm{mg} / \mathrm{kg})$ of DA-9701 on the upper GI transit was tested in the OIBD model.

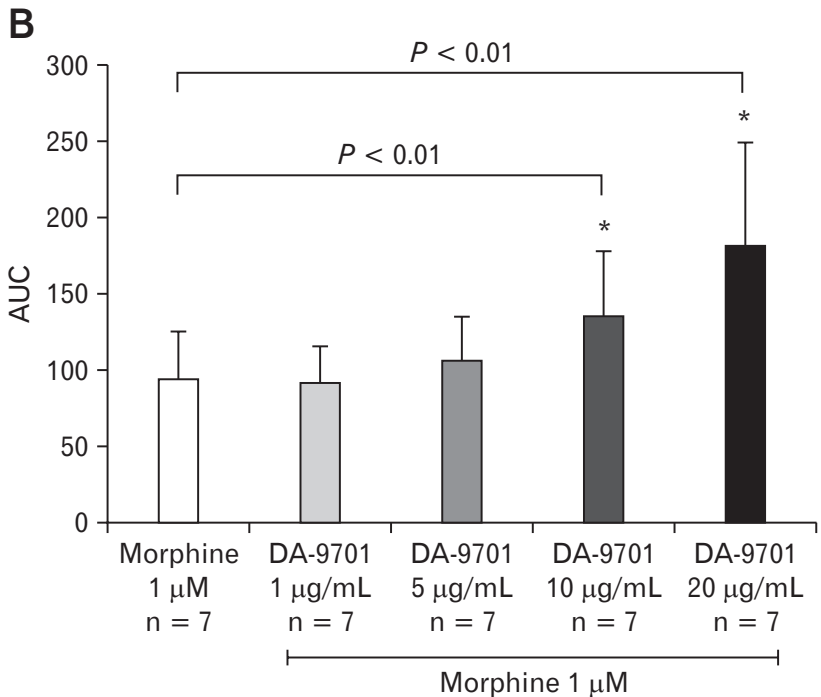

Figure 7. Effect of DA-9701 on morphine treated ileal muscle. DA-9701 increased maximal amplitude and under the curve (AUC) at concentrations of $20 \mu \mathrm{g} / \mathrm{mL}$. (A) Percent change of amplitude from morphine only ( $\left.{ }^{*} P=0.010\right)$. (B) Percent change of AUC from morphine only $\left({ }^{\star} P=\right.$ $0.010)$. Values are represented in mean \pm SEM.
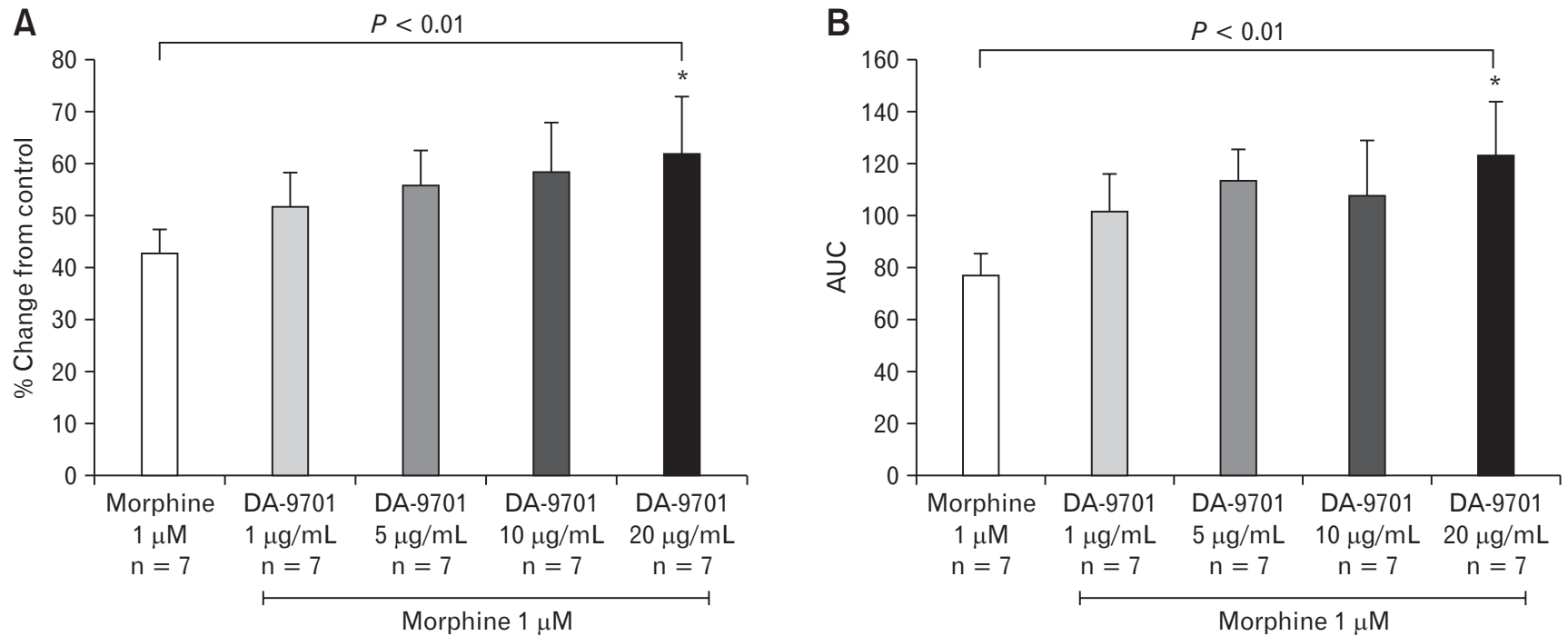

Figure 8. Effect of DA-9701 on morphine treated distal colon muscle. DA-9701 increased maximal amplitude and under the curve (AUC) at concentrations of $20 \mu \mathrm{g} \mathrm{mL}{ }^{-1}$. (A) Percent change of amplitude from morphine only ( ${ }^{*} P=0.01$ ). (B) Percent change of AUC from morphine only ( ${ }^{*} P$ $=0.01)$. Values are represented in mean \pm SEM. 
As shown in Figure 9, the administration of $3 \mathrm{mg} / \mathrm{kg}$ and $10 \mathrm{mg} /$ $\mathrm{kg}$ of DA-9701 in morphine induced OIBD cases significantly increased the charcoal transit $(12.72 \pm 13.21$ vs $24.99 \pm 8.20)$ and (12.72 \pm 13.21 vs $23.25 \pm 12.52$ ), respectively (Fig. 9). Hence, DA-9701 significantly reversed abnormally delayed upper GI transit induced by morphine.

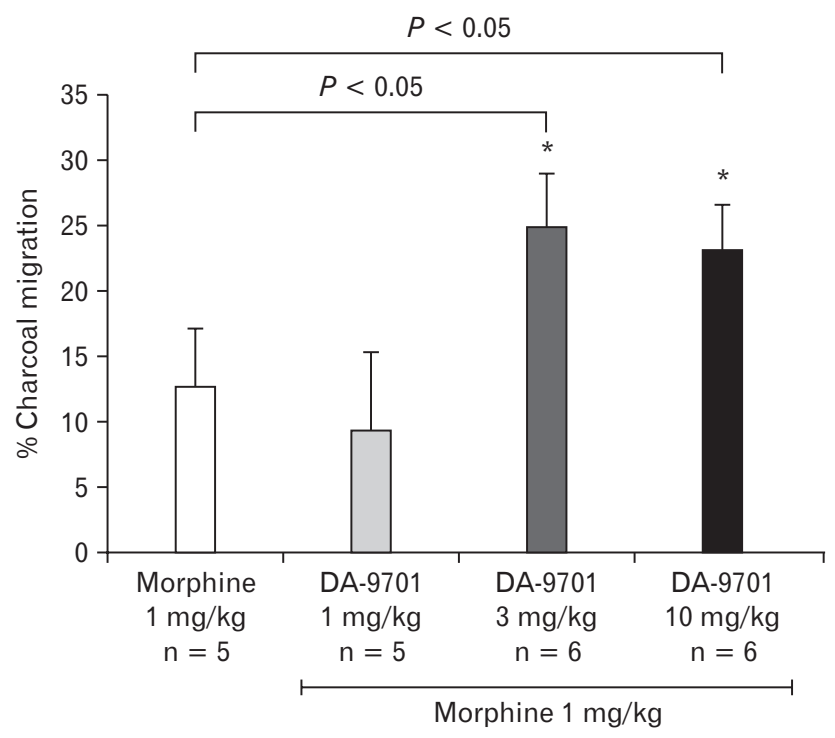

Figure 9. The effect of DA-9701 on upper gastrointestinal transit in opioid induced bowel dysfunction guinea pig model. Values are mean \pm SEM. ${ }^{*} P<0.05$ and ${ }^{*} P<0.01$ in comparison to morphine only.

\section{Effects of DA-9701 on Fecal Expulsion in Opioid Induced Bowel Dysfunction Model}

In the fecal expulsion assay the most effective dose of morphine $(0.3 \mathrm{mg} / \mathrm{kg})$ was selected to induce the experimental model of the OIBD in the guinea pig. The prokinetic effect of different doses $(1,3$, and $10 \mathrm{mg} / \mathrm{kg})$ of DA-9701 on the lower GI transit was tested in the OIBD model. As shown in Figure 10, the cumulative fecal pellet output and cumulative fecal pellet output weight (g) significantly increased in presence of all 3 doses of DA-9701. These results showed that DA-9701 significantly restored lower GI transit induced by morphine.

\section{Discussion}

Opioid induced bowel dysfunction (OIBD) is associated with adverse effect of opioid analgesics on $\mu$-opioid receptors located in the enteric nervous system. Opioid activated $\mu$-receptors disrupt GI function that leads to delayed gastric emptying, infrequent bowel movements and difficulty in rectal expulsion of stool. Webster et $\mathrm{al}^{21}$ hypothesized the action of alvimopan as selective antagonism of peripheral $\mu$-opioid receptors that leads to restoration of GI function and relieve opioid induced bowel dysfunction. Most of the studies suggested the activation of opioid receptors in the GI tract as a major cause of OIBD. ${ }^{8,9}$ Several prokinetic agents have been developed recently, yet their use is restricted due to serious side ef-

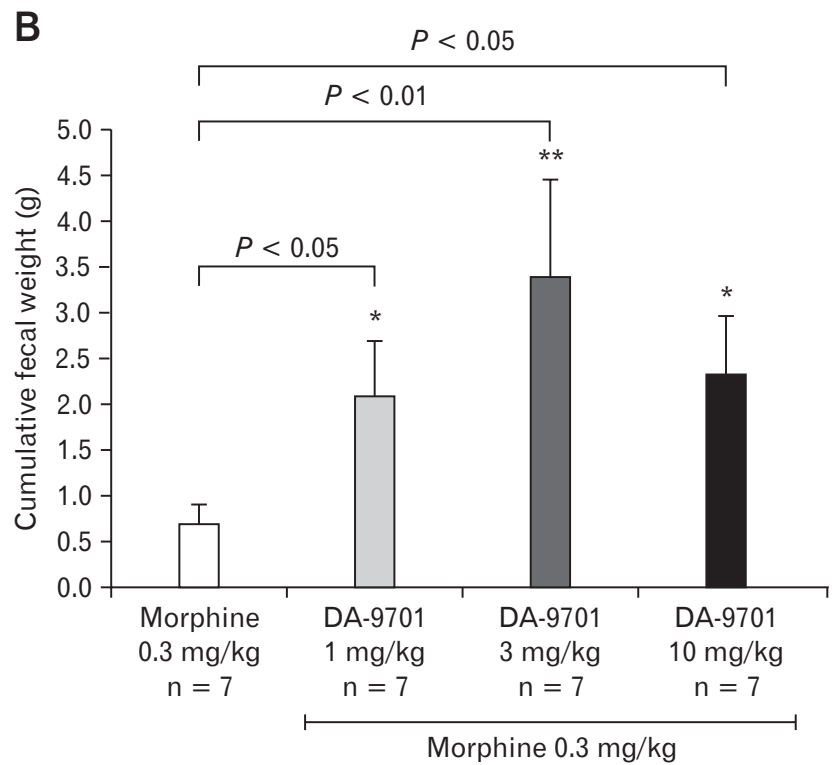

Figure 10. Effect of DA-9701 on (A) cumulative fecal pellet number and (B) cumulative fecal weight mean \pm SEM ( $\mathrm{n}=6 / \mathrm{group})$; ${ }^{*} P<0.05$ and ${ }^{*} P<0.01$ in comparison to morphine only. 
fects and failure to confirm their efficacy. On the other hand Sloots et $\mathrm{al}^{22}$ studied the efficacy and safety of prucalopride in chronic constipation from opioid induced constipation, and they suggested improved bowel movement frequency. Hence, there is an increasing challenge to develop safer and more effective therapeutic agents for the treatment of OIBD. In this series, we have tested a newly formulated prokinetic agent called DA-9701, and its pharmacological effects on OIBD induced GI motor dysfunction. Our current study clearly demonstrated the promising effects of DA-9701 on enhancing GI motor function as evaluated by ileal and distal colon muscle contraction and GI transit. DA-9701 augments the amplitude of contraction of normal ileal muscle. The amplitude of contraction of the distal colon muscle was not significantly increased as that of the ileum. This suggests that the prokinetic effect of DA-9701 on the small intestine was greater than that of the distal large intestine. Our findings corroborate with a previous animal study in which DA-9701 significantly accelerated gastric emptying in normal rats, as well as in abnormally-induced conditions such as delayed gastric emptying caused by apomorphine, a dopamine agonist. ${ }^{14}$ Furthermore, DA-9701 also significantly improved gastric compliance in canines. $^{15}$

Our study showed that morphine induces differential dose dependent inhibition of the upper and lower GI motility. The differential action of morphine in the GI tract can be partially explained by opioid receptor distribution, receptor density, and tissue specific localization. Hence, we speculate major differences in opioid receptor distribution within the upper and lower GI tract, and hence morphine can act differently in receptor binding, opioid specific signal transduction pathways and receptor desensitization. More studies are warranted in order to ascertain the distribution and exact localization of the opioid receptors. Additionally, the effect of DA9701 on GI transit in morphine OIBD was determined by charcoal transit and fecal pellet output assays. The oral administration of DA-9701 in the preclinical OIBD model, significantly reversed the GI inhibitory effects of morphine and restored the delayed upper and lower GI transit. These result showed the remarkable prokinetic properties of DA-9701 that could enhance GI motility both in normal and abnormal conditions. Therefore, the pharmacological action and affinity of DA-9701 need to be addressed in OIBD.

The novel prokinetic agent DA-9701 exerts a prokinetic effect in the OIBD model of the guinea pig. It clearly increased the ileal and distal colon muscle contraction and improved gastrointestinal transit in OIBD. This drug may serve as a useful candidate drug in a clinical trial for OIBD.
Financial support: This study was sponsored by Dong-A Science and Technology, Seoul, Korea.

\section{Conflicts of interest: None.}

Author contributions: Zahid Hussain was responsible for data analysis and drafting of the manuscript; Young Ju Lee was in charge of guinea pig handling, experimental design; Kwang Won Rhee was responsible for data analysis and critical revision of the manuscript; and Hyojin Park was the corresponding author, and was responsible for study concept, design, critical revision, and study supervision.

\section{References}

1. Bianchi G, Ferretti P, Recchia M, Rocchetti M, Tavani A, Manara L. Morphine tissue levels and reduction of gastrointestinal transit in rats. Correlation supports primary action site in the gut. Gastroenterology 1983;85:852-858

2. Kurz A, Sessler DI. Opioid-induced bowel dysfunction: pathophysiology and potential new therapies. Drugs 2003;63:649-671.

3. Bell TJ, Panchal SJ, Miaskowski C, Bolge SC, Milanova T, Williamson R. The prevalence, severity, and impact of opioid-induced bowel dysfunction: results of a US and European patient survey (PROBE 1). Pain Med 2009;10:35-42.

4. Panchal SJ, Müller-Schwefe P, Wurzelmann JI. Opioid-induced bowel dysfunction: prevalence, pathophysiology and burden. Int J Clin Pract 2007;61:1181-1187.

5. Holzer P. Treatment of opioid-induced gut dysfunction. Expert Opin Investig Drugs 2007;16:181-194.

6. Reimer K, Hopp M, Zenz M, et al. Meeting the challenges of opioidinduced constipation in chronic pain management - a novel approach. Pharmacology 2009;83:10-17.

7. Davis MP. The opioid bowel syndrome: a review of pathophysiology and treatment. J Opioid Manag 2005;1:153-161.

8. De Luca A, Coupar IM. Insights into opioid action in the intestinal tract. Pharmacol Ther 1996;69:103-115.

9. Wood JD, Galligan JJ. Function of opioids in the enteric nervous system. Neurogastroenterol Motil 2004;16(suppl 2):17-28.

10. Holzer P. Opioids and opioid receptors in the enteric nervous system from a problem in opioid analgesia to a possible new prokinetic therapy in humans. Neurosci Lett 2004;361:192-195.

11. Fei G, Raehal K, Liu S, et al. Lubiprostone reverses the inhibitory action of morphine on intestinal secretion in guinea pig and mouse. J Pharmacol Exp Ther 2010;334:333-340.

12. Pappagallo M. Incidence, prevalence, and management of opioid bowel dysfunction. Am J Surg 2001;182(5A suppl):11S-18S.

13. Jung YS, Kim MY, Lee HS, Park SL, Lee KJ. Effect of DA-9701, a novel prokinetic agent, on stress-induced delayed gastric emptying and hormonal changes in rats. Neurogastroenterol Motil 2013;25:254-259, 
e166.

14. Lee TH, Choi JJ, Kim DH, et al. Gastroprokinetic effects of DA-9701, a new prokinetic agent formulated with Pharbitis Semen and Corydalis Tuber. Phytomedicine 2008;15:836-843.

15. Kim ER, Min BH, Lee SO, Lee TH, Son M, Rhee PL. Effects of DA-9701, a novel prokinetic agent, on gastric accommodation in conscious dogs. J Gastroenterol Hepatol 2012;27:766-772.

16. Lim CH, Choi MG, Park H, Baeg MK, Park JM. Effect of DA-9701 on gastric emptying in a mouse model: assessment by ${ }^{13} \mathrm{C}$-octanoic acid acid breath test. World J Gastroenterol 2013;19:4380-4385.

17. Lee TH, Son M, Kim SY. Effects of corydaline from Corydalis tuber on gastric motor function in an animal model. Biol Pharm Bull 2010;33:958-962.

18. Hung TM, Na M, Dat NT, et al. Cholinesterase inhibitory and antiamnesic activity of alkaloids from Corydalis turtschaninovii. J Ethnophar- macol 2008;119:74-80.

19. Hung TM, Ngoc TM, Youn UJ, et al. Anti-amnestic activity of pseudocoptisine from Corydalis Tuber. Biol Pharm Bull 2008;31:159-162.

20. Lee TH, Kim KH, Lee SO, Lee KR, Son M, Jin M. Tetrahydroberberine, an isoquinoline alkaloid isolated from corydalis tuber, enhances gastrointestinal motor function. J Pharmacol Exp Ther 2011;338:917924.

21. Webster L, Jansen JP, Peppin J, et al. Alvimopan, a peripherally acting mu-opioid receptor (PAM-OR) antagonist for the treatment of opioidinduced bowel dysfunction: results from a randomized, double-blind, placebo-controlled, dose-finding study in subjects taking opioids for chronic non-cancer pain. Pain 2008;137:428-440.

22. Sloots CE, Rykx A, Cools M, Kerstens R, De Pauw M. Efficacy and safety of prucalopride in patients with chronic noncancer pain suffering from opioid-induced constipation. Dig Dis Sci 2010;55:2912-2921. 